Recepción: 27 / 12 / 2017

Aceptación: 16 / 02 / 2018

Publicación: 08 / 05 / 2018

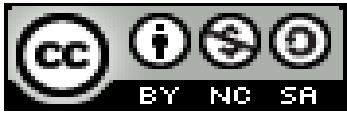

Ciencias de la salud

Artículo de Revisión

\title{
Características de traumatismo dental en niños de 5-13 años de edad
}

\section{Characteristics of dental trauma in children 5-13 years of age}

\section{Características do traumatismo dentário em crianças de 5 a 13 anos de idade}

\author{
Juan A. Oliveira-del Río ${ }^{\mathrm{I}}$ \\ juanoliveiradelrio@hotmail.com
}

Alcira M. Alvarado-Solórzano II alcira.alvarado@gmail.com

Betty E. Guanotoa-Lincango ${ }^{\text {III }}$ betty.guanotoa@gmail.com

Correspondencia: juanoliveiradelrio@hotmail.com

\footnotetext{
${ }^{\text {I }}$ Magister Scientiarum en Odontopediatria, Doctor en Ciencias Odontológicas, Odontólogo, Docente de la Universidad Laica "Eloy Alfaro” de Manabí. Manta, Ecuador.

${ }^{\text {II }}$ Magister en Gerencia y Auditoría en Salud Bucal, Residente del post-grado de Odontopediatría de la Universidad de Carabobo, Docente de la Universidad Laica "Eloy Alfaro” de Manabí, Manta, Ecuador.

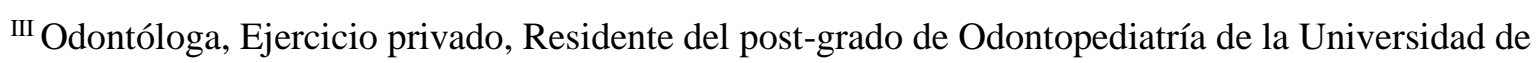
Carabobo, Carabobo, Venezuela.
} 


\title{
Resumen
}

En la actualidad los traumatismos dentales, son la segunda causa de demanda de atención dental en niños tras la caries dental. Se realizó un estudio observacional descriptivo, de corte transversal, con la finalidad de determinar las características epidemiológicas de los traumatismos dentales en niños de 5 a 13 años de edad. La población de estudio estuvo conformada por 52 niños, que acudieron a consulta diagnosticados con traumatismos dentales. Entre las principales variables analizadas figuraron: edad, sexo, factores predisponentes y desencadenantes y tipo de trauma, respetándose la ética médica. En los resultados, predominó el sexo masculino entre 8 y 10 años de edad, siendo el resalte aumentado y la incompetencia bilabial los factores predisponentes con mayor prevalencia, donde la fractura no complicada de corona fue la lesión más marcada, desencadenados por la práctica de deportes. Los traumatismos dentales fueron considerados una situación de urgencia a diagnosticar y tratar de forma rápida y certera por el odontólogo.

Palabras clave: traumatismo; factores desencadenantes y predisponentes.

\begin{abstract}
Currently, dental trauma is the second cause of demand for dental care in children after dental caries. A descriptive, cross-sectional observational study was carried out in order to determine the epidemiological characteristics of dental trauma in children from 5 to 13 years of age. The study population consisted of 52 children, who attended the consultation diagnosed with dental trauma. Among the main variables analyzed were: age, sex, predisposing factors and triggers and type of trauma, respecting medical ethics. In the results, the male sex between 8 and 10 years of age predominated, with the prominence increased and bilabial incompetence the predisposing factors with higher prevalence, where the uncomplicated fracture of the crown was the most marked injury, triggered by the practice of sports. Dental trauma was considered an emergency situation to diagnose and treat quickly and accurately by the dentist.
\end{abstract}

Keywords: trauma; triggering and predisposing factors.

\section{Resumo}

Atualmente, o trauma dentário é a segunda causa de demanda por atendimento odontológico em crianças após a cárie dentária. Foi realizado um estudo observacional descritivo, transversal, com o objetivo de determinar as características epidemiológicas do traumatismo dentário em crianças 
de 5 a 13 anos de idade. A população do estudo foi composta por 52 crianças, que compareceram à consulta com diagnóstico de traumatismo dentário. Entre as principais variáveis analisadas estavam: idade, sexo, fatores predisponentes e fatores desencadeantes e tipo de trauma, respeitando a ética médica. Nos resultados, uma predominância de homens entre 8 e 10 anos, com o aumento da projeção e incompetência bilabial fatores predisponentes mais prevalente onde descomplicada coroa fratura foi mais acentuada lesão, desencadeada por esportes. $\mathrm{O}$ traumatismo dentário foi considerado uma situação de emergência para diagnosticar e tratar de forma rápida e precisa pelo dentista.

Palavras chave: trauma; fatores desencadeantes e predisponentes.

\section{Introducción}

Los traumatismos dentarios son accidentes muy frecuentes entre la población infantil y juvenil, con una prevalencia que varía entre un 10.7 y un $43.8 \%$, dependiendo de la edad, el sexo y la población seleccionada, y constituyen una de las experiencias más desagradables para el niño y sus padres. Además, en los últimos años han experimentado un incremento considerable, en paralelo con otro tipo de accidentes. ${ }^{1-2-3}$

Son más frecuentes en niños que en niñas en una proporción 2:1. El pico de incidencia en dentición temporal abarca de los 2 a los 4 años y en dentición permanente de los 8 a los 10 años. ${ }^{4}$

La mayoría de los traumatismos dentales en dientes permanentes pueden ser tratados con éxito. La rapidez con que se lleve a cabo el tratamiento de urgencia es fundamental para prevenir la aparición de complicaciones, 6 por lo que el pronóstico depende de la inmediatez de su atención, sin restarle importancia a la individualidad de cada paciente, así como del nivel cultural de este y sus familiares. ${ }^{5}$

Uno de los objetivos fundamentales de la odontología es la conservación de los tejidos dentales, el cual está amenazado por las lesiones traumáticas producidas en los dientes y sus tejidos de sostén, que a menudo terminan con la pérdida de los mismos. ${ }^{6}$

Los estudios epidemiológicos revelan que el trauma dentario constituye un serio problema de salud que se acompaña tanto de daños estéticos y psicológicos, como sociales. Pueden variar desde simples fracturas de esmalte hasta avulsiones, y pueden observarse diferentes tipos de 
lesiones: fracturas óseas, lesiones de tejidos blandos, fracturas dentales o lesiones de los tejidos periodontales conocidas como luxaciones o desplazamientos.$^{7}$

Por su elevada prevalencia e incidencia y repercusión en el estado de salud bucal, es de gran importancia para los estomatólogos y población en general identificar los factores predisponentes de traumatismos dentarios, con la finalidad de realizar actividades preventivas que contribuyan a disminuir su aparición, motivo por el cual se diseñó esta investigación.

\section{Metodología}

Se realizó un estudio observacional descriptivo, de corte transversal, en niños de 5- 13 años de edad que asistieron a consulta de odontopediatria en el segundo semestre del 2017, con la finalidad determinar las características epidemiológicas de los traumatismos dentales.

La población de estudio estuvo conformada por todos aquellos niños que acudieron a consulta, diagnosticados con traumatismos dentales (52).

Entre las principales variables analizadas figuraron: edad, sexo, factores predisponentes y desencadenantes y tipo de trauma, respetándose la ética médica.

Entre los factores predisponente resalta: distancia tomada con regla milimetrada desde el borde incisal cara palatina del incisivo superior ya sea derecho o izquierdo, hasta el borde incisal cara vestibular pero del homólogo inferior) e incompetencia bilabial (cuando el paciente en posición de reposo posee sus labios separados y para lograr el cierre provoca contracción de la musculatura (músculo orbicular de los labios y músculo mentoniano); factores desencadenantes: práctica de deporte (relación de actividades deportivas con carácter masivo (en equipos), voleibol, pelota, fútbol y baloncesto); accidentes (accidentes automovilísticos (de tránsito) y caídas (caídas en juegos, caídas contra objetos al caminar, caídas de escaleras, caídas de la cama, entre otras).

Traumatismos dentarios: según clasificación Método de Ingeborg Jacobsen ${ }^{8}$

- Infractura del esmalte

- Fractura no complicada de la corona

- Factura complicada de la corona 
- Fractura de corona y raíz

- Fractura de raíz

- Concusión

- Luxación

- Exarticulación o avulsión

Para la recogida de datos se elaboró un instrumento, que se completó a partir de la entrevista, la observación e inspección de la cavidad bucal. Para la introducción de los datos se confeccionó una base de datos en la aplicación Microsoft Excel que fue procesada con el paquete estadístico SPSS versión 8.0, mediante el cual se estimaron las medidas de resumen para variables cualitativas: frecuencias absolutas y porcentajes.

\section{Resultados}

Del total de pacientes atendidos los grupos de edades más afectados fueron de 8-10 y 11-14 (46.6\%), siendo significativo el predominio en el sexo masculino (67.3\%). (Tabla 1)

Tabla 1. Total de pacientes atendidos

\begin{tabular}{|l|l|l|l|l|l|l|}
\hline \multirow{2}{*}{$\begin{array}{c}\text { Grupos de } \\
\text { edades }\end{array}$} & \multicolumn{2}{|c|}{ Masculino } & \multicolumn{2}{c|}{ Femenino } & \multicolumn{2}{c|}{ Total } \\
\cline { 2 - 7 } & No & \% & \multicolumn{1}{c|}{ No } & \% & No & \% \\
\hline $5-7$ & 3 & 8.5 & 1 & 5.8 & 4 & 7.6 \\
\hline $8-10$ & 19 & 54.2 & 5 & 29.4 & 24 & 46.6 \\
\hline $11-13$ & 13 & 37.1 & 11 & 64.7 & 24 & 46.6 \\
\hline Total & 35 & 67.3 & 17 & 32.6 & 52 & 100 \\
\hline
\end{tabular}

Del total de pacientes afectados con traumatismos dentales, el 78.0\% presentó resalte aumentado y $17.0 \%$ con incompetencia bilabial, siendo más afectadas las edades de 8-10 años, con un $50.0 \%$. (Tabla 2) 
Tabla 2. Factores predisponente según grupo de edad

\begin{tabular}{|l|l|l|c|c|c|l|}
\hline \multirow{2}{*}{\begin{tabular}{c} 
Grupos $\begin{array}{c}\text { Fe } \\
\text { edades }\end{array}$ \\
\cline { 2 - 8 }
\end{tabular}} & \multicolumn{6}{|c|}{ Ractores predisponente } \\
\cline { 2 - 8 } & \multicolumn{2}{|c|}{ No } & $\%$ & \multicolumn{2}{c|}{$\begin{array}{c}\text { No } \\
\text { bilabial }\end{array}$} & \multicolumn{2}{c|}{ Total } \\
\hline $5-7$ & 1 & 3.1 & 2 & 28.5 & 3 & 7.3 \\
\hline $8-10$ & 16 & 50.0 & 2 & 28.5 & 18 & 43.9 \\
\hline $11-13$ & 15 & 46.8 & 5 & 71.4 & 20 & 48.7 \\
\hline Total & 32 & 78.0 & 7 & 17.0 & 41 & 100 \\
\hline
\end{tabular}

Por otra pare los factores desencadenantes fueron más frecuentes en las edades de 11-13 años con $50.0 \%$, siendo la práctica de deportes el más frecuente.

En relación con los hábitos bucales deformantes se obtuvo que la succión digital y la respiración bucal se manifestó en el $61.5 \%$ y el $48.0 \%$ respectivamente

Tabla 3. Distribución de los pacientes según hábitos bucales deformantes.

\begin{tabular}{|l|c|c|}
\hline \multicolumn{1}{|c|}{$\begin{array}{c}\text { Hábitos bucales } \\
\text { deformantes }\end{array}$} & No & \% \\
\hline Succión digital & 32 & 61.5 \\
\hline Lengua protráctil & 18 & 34.6 \\
\hline Onicofagia & 8 & 15.3 \\
\hline Respirador bucal & 25 & 48.0 \\
\hline Queilofagia & 6 & 11.5 \\
\hline
\end{tabular}

La mayoría de los traumatismos fueron fractura no complicada de la corona $73.3 \%$ le continuaron: concusión, luxación y avulsión. 


\section{Discusión}

Respecto al sexo y edad, coincidimos con resultados que son similares a los encontrados por varios autores, quienes plantean la predilección por el sexo masculino. Puede ser debido a que los varones practican deportes y juegos violentos con mayor intensidad y frecuencia que las hembras. ${ }^{9-10}$

La mayoría de los investigadores demuestran que los varones sufren al menos dos veces más lesiones en la dentición permanente que las hembras, factor que está, sin dudas, relacionado con su participación más activa e intensa en juegos y deportes. Esta preponderancia masculina no es tan marcada en la dentición temporal, aunque algunos señalan, que inclusive en los prescolares, los varones accidentados son más que las niñas. ${ }^{11}$

Estudios realizados por diferentes autores, consideran que la incidencia de lesiones por traumas se incrementa con el propio desarrollo psicomotor del niño, de forma geométrica y en proporciones, en ocasiones alarmantes, considerándose que existen edades donde el auge de la actividad humana de los pequeños propicia prácticamente el 50\% de estas lesiones. ${ }^{12}$

Los resultados de esta investigación no concuerdan con la realizada por Torralbas ${ }^{13}$ la cual obtiene que, de la totalidad de pacientes afectados por lesiones traumáticas, el mayor porcentaje se encuentra en el grupo de 9-10 años con el 46,1\% ni con los Sánchez. ${ }^{14}$

Se conoce que los hábitos deformantes de larga duración alteran la función y equilibrio normal de los dientes y maxilares. Los hábitos de presión interfieren en el crecimiento normal y en la función de la musculatura orofacial. ${ }^{15}$

La succión digital es uno de los hábitos más frecuentes en la infancia y se relaciona con la maloclusión, particularmente con resalte aumentado incisal ${ }^{16}$

El hábito bucal deformante más frecuente fue la succión digital. Estos resultados coinciden con Maya, ${ }^{17}$ que describe como hábito deformante más frecuente a la succión digital en su investigación. Otros estudios sostienen que la presencia de hábitos deformantes, constituyen factores predisponentes al trauma, por eso se considera de vital importancia la detección temprana y el control de estos hábitos, por parte del estomatólogo general integral ${ }^{18}$ 
El factor desencadenante de mayor frecuencia lo constituyó la práctica de deportes. Al respecto los autores argumentan que los niños que practican actividades deportivas sistemáticas están en contacto permanente con factores de riesgo, por lo que tienen mayor probabilidad de fracturarse los dientes. Coincidiendo con los resultados de González García1 et al. ${ }^{11}$

Hacer prevención es la labor principal y más importante, pero si ya el hecho de un traumatismo de los dientes está consumado, por leve que pueda aparecer la lesión, se impone acudir de inmediato al odontólogo y recibir la debida valoración y el tratamiento adecuado. No debe olvidarse que un dejarlo para después pudiera convertirse en un hecho fatal para la vida de los dientes.

Finalmente, en el estudio predominó el sexo masculino entre 8 y 10 años de edad, siendo el resalte aumentado y la incompetencia bilabial los factores predisponentes con mayor prevalencia, donde la fractura no complicada de corona fue la lesión más marcada, desencadenados por la práctica de deportes. Los traumatismos dentales fueron considerados una situación de urgencia a diagnosticar y tratar de forma rápida y certera por el odontólogo.

\section{Referencias bibliográficas}

1. González S.Traumatismos dentales.RCOE. 2003;8:125-6.

2. Andreasen JO. Buonocore memorial lecture. Adhesive dentistry applied to the treatment of traumatic dental injuries. Oper Dent. 2001;26:328-35.

3. Paredes V, paredes C. Traumatismos dentarios en la infancia. An Pediatr Contin. 2005;3(6):375-8.

4. Dorney B. Inappropriate treatment of traumatic dental injuries. Aust Endod J. 1999;25:768.

5. Yero Mier IM, González Fortes B, Mursulí Sosa M, Cruz Milián MC. Traumatismo dentario. 15 a 18 años. Escuela Militar Camilo Cienfuegos. Sancti Spíritus 2011. Gac Méd Espirit. 2013 [citado 2017 Dic 11]; 15(1). Disponible en: http://scielo.sld.cu/scielo.php?script=sci_arttext\&pid=S1608 -89212013000100012\&lng= 
6. Ramírez Acosta Y, Verdecia Moreno A, Correa Acosta D, Galán Gutierrez E, Rodríguez del Toro M. Traumatismo dentario en atletas santiagueros de alto rendimiento. MEDISAN. 2014 [citado 2017 Sept 13]; 18(8). Disponible en: http://scielo.sld.cu/scielo.php?script=sci_arttext\&pid=S1029 $-30192014000800003 \& \operatorname{lng}=\mathrm{es}$

7. Garrido Andreu MI, Sardiña Alayón S, Gispert Abreu E, Valdés García P, Legón Pandilla $\mathrm{N}$, Fuentes Balido J, et al. Guías prácticas clínicas ante traumatismos dentarios y faciales. En: Guías Prácticas de Estomatología. La Habana: Ciencias Médicas; 2003.p.128-152.

8. Malagón Mesa E, Vento Tejera NM, Gómez Muñiz MI, Díaz Llana CZ. Comportamiento clínico epidemiológico de los traumatismos dentales en escuelas urbanas de San Juan y Martínez. Rev Cienc Méd. 2013 [citado 11 Sept 2017]; 17(2). Disponible en: http://scielo.sld.cu/scielo.php?pid=S1561 -31942013000200009\&script=sci_arttext

9. González Martínez C, Rivero Villalón M, Pérez Navarro N, Capdevila Gort SE. Traumatismos dentarios en dentición permanente en el servicio de urgencias estomatológicas de Melena del Sur. Rev de Ciencias Médicas de la Habana. 2015 [citado 2017 Sept 11]- 21(2): [Aprox. 11 p.]. Disponible en: http://revcmhabana.sld.cu/index.php/rcmh/rt /printerFriendly/787/1229

10. González García X, Crespo Sánchez M, Cardentey García J, Porras Mijans O. Traumatismos dentales en niños de 7 a 11 años. Rev. Ciencias Médicas de Pinar del Río. Julioagosto, 2016; 20 (4):465-471.

11. Torres Silva María del Carmen, Barberán Díaz Yoannys, Bruzón Díaz Ana Maité, Jorge Figueredo Elizabeth, Rosales García Yuniel. Factores predisponentes de trauma dental en escolares del municipio Rafael Freyre. ccm. 2017 Sep [citado 2017 Sep 19]; 21(3): 798-808. Disponible en: http://scielo.sld.cu/scielo.php?script=sci_arttext\&pid=S1560$43812017000300016 \& \operatorname{lng}=\mathrm{es}$.

12. Valdés Álvarez R, Montero del Castillo ME, Cabañas Lores C, González Naya G, Legón Padilla N, Nodarse Rodríguez $\mathrm{M}$, et al. Lesiones traumáticas de los dientes en niños y adolescentes. En: Manual de Odontopediatría. La Habana: Ciencias Médicas; 2014 [citado 9 sep 
2017];

[142p.].

Disponible

en:

http://www.bvs.sld.cu/libros/manual_odontopediatria/manual_odontopediatria_completo.pdf

13. Torralbas Ezpeleta L, Pérez García MC, Valverde Fernández I. Traumatismos dentarios en niños de escuelas primarias urbanas pertenecientes a la Clínica Estomatológica Manuel Angulo Farrán. CCM. 2013. [citado 8 sep 2017]; 17(1 Supl 1). Disponible en: http://www.revcocmed.sld.cu/index.php/cocmed/article/view/1274

14. Sánchez Barrio PG, Sánchez Santos L, Pérez Piñeiro CJ, de la Torre Rodríguez E. Factores predisponentes del trauma dental, Escuela Primaria "República de Angola" (20122013).Rev Cubana Estomatol. 2015[citado 8 sep 2017]; 52(2). Disponible en: http://www.revestomatologia.sld.cu/index.php/est/article/view/747/130

15. Aguilar M, Villaizá C, Nieto I. Frecuencia de hábitos orales, factor etiológico de maloclusiones en población escolar. Rev Latinoam Ortod Odontopediatr . 2009 [citado 10 Mar 2013]. Disponible en:http://www.ortodoncia.ws/publicaciones/2009art22.as

16. Medina C. Prevalencia de maloclusiones dentales en un grupo de pacientes pediátrico. Acta odontol. venez 2010; 48(1).

17. Maya Hernández B. Efectividad de la persuasión como terapia para la eliminación de hábitos bucales incorrectos. Provincia de Cienfuegos. Rev Cubana Ortod. [En línea]. 2009 [Consultado: 3 sept 2017];15(2):66-70. Disponible en: http://www.bvs.sld.cu/revistas/ord/vol15_2_00/ord04200.htm

18. Rodríguez Soto A, Pérez Cordero Y, Fernández Collazo ME, Vila Morales D, Cuevillas Guerra G. Traumas dentoalveolares relacionados con maloclusiones en menores de 15 años. Revista Cubana Estomatol. 2011 [Citado 3 sept 2017]; 48(3): 241-8. Disponible en: http://scielo.sld.cu/scielo.php?script=sci_arttext\&pid=S0034-75072011000300006\&lng=es 